Military Technical College, Kobry El-Kobbah, Cairo, Egypt

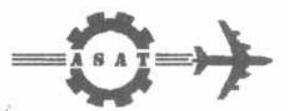

$9^{\text {th }}$ International Conference

On Aerospace Sciences \&

Aviation Technology

\title{
PROBLEMS ASSOCIATED WITH DESIGNING STRIPLINE RESONATOR FOR WIDE BAND MATERIAL CHARACTERIZATION
}

\author{
Abd El-Azeem M.H*
}

\begin{abstract}
The electrical properties of the dielectric materials can be described in terms of their relative dielectric constant and dielectric loss tangent. To measure these electrical properties at microwave frequencies, the most commonly used technique is the resonant technique. This technique relates the measured resonant frequency and the quality factor of a resonator to the characteristic of the material from which it was constructed. The coplaner coupled stripline resonator is a modified version of the stripline resonator to increase the accuracy of measurements.

This paper presents an analysis of the coplaner coupled stripline resonator technique as will as the proper design of it's gap, a Pspice model of this gap, and finally presents a discussion of the capacitance behavior of this gap above some certain frequencies depending on the dimensions of the gap.
\end{abstract}

\section{KEY WORDS}

Microstrip, Striplines, Resonators.

\section{COPLANER STRIPLINE RESONATOR CONFIGURATION}

The coplaner coupled stripline resonator (CCSLR) configuration is fundamentally the same as that of the stripline resonator (SLR). The main difference is the replacement of the two striplines coupling sections by coplanar line sections as shown in figures (1), (2)

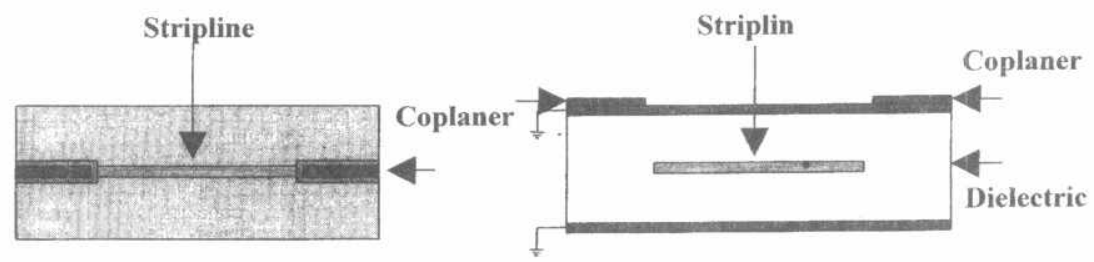

Fig. (1). (2) Coplaner Coupled Stripline Resonator

*Egyption Armed Forces 
As shown in the figures, the middle stripline resonator is now coupled via two coplanar line sections at either end. The coplanar line sections are fabricated on the top surface of the structure. Coupling is determined by the overlap (or spacing) between the edges of the coplanar lines and the stripline sides.

\section{COPLANER COUPLED STRIPLINE RESONATOR TECHNIQUE}

The analysis presented in this section is equally and valid for both the conventional stripline configuration and the coplaner coupled stripline resonator since it is related the resonator section (which is a stripline in both cases). Using frequency domain measurement, (e.g. using a vector network analyzer), we can calculate the relative permitivity of the dielectric from the output resonance frequencies of the stripline resonator. Assuming $\lambda \mathrm{g}$ is the wavelength in the stripline at the frequency $\mathrm{fr}$, we can write the following relation in accordance to the theory of stripline,

$\lambda_{\mathrm{g}}=\frac{\lambda_{0}}{\sqrt{\varepsilon_{\mathrm{r}}}}$

where:

$\lambda_{0} \ldots$ is the wavelength of the wave with frequency fr in free space,

$\lambda_{0}=\frac{c}{f_{r}}$

When the length $L$ of the stripline section is the integer multiples of $\frac{\lambda g}{2}$, the line becomes resonant. At this time, the output signal of the resonator achieves its maximum value. Hence, the resonant condition for a line resonator can be written as,

$\mathbf{L}=\mathbf{n} \cdot \frac{\lambda_{\mathrm{g}}}{2}$

From equations (1), (2), and (3) we can write the relation between the measured resonance frequencies of the stripline resonator, fr and the relative permitivity of the used dielectric in this resonator, ${ }_{r}$ as follows,

$\varepsilon_{r}=\left(\frac{c}{2 \frac{f_{r}}{n} L}\right)^{2}$

where

C ... is the speed of the light in free space. 
$f_{r} \ldots$ is the resonant frequency.

$\varepsilon_{r} \ldots$ is the relative permittivity.

$L$... is the center strip length.

$n=1,2,3 \ldots$ is the order of resonance.

Neglecting the radiation terms, the quality factor $\mathrm{Q}_{0}$ of a low loss stripline resonator can be expressed as:

$\frac{1}{Q_{0}}=\frac{1}{Q_{c}}+\frac{1}{Q_{d}}$

where

$Q_{c} \ldots$ is the term due to loss in the conductor

$Q_{d} \ldots$ is the term due to the dielectric loss tangent

For lines with small attenuation constant $\alpha$, the quality factor of the line resonator can be related to $\alpha$ as:

$\mathrm{Q}=\frac{\pi \sqrt{\varepsilon_{\mathrm{r}}}}{\alpha \lambda}$

The dielectric attenuation constant ${ }^{\alpha_{d}}$ is related to the dielectric loss tangent $\tan \delta$ as follows:

$\alpha_{d}=\frac{\pi \sqrt{\varepsilon_{r}}}{\lambda_{0}} \quad \tan \delta$

Substituting by (7) in (6) gives

$\frac{1}{Q_{d}}=\tan \delta$

Using the same equation (6), we can determine the conductor loss term if $\alpha_{c}$ (attenuation due to conductor loss) is known as follows. 
$\frac{1}{\mathrm{Q}_{\mathrm{c}}}=\frac{1.5911}{\sqrt{\sigma \mathrm{f}}} \mathrm{g}(\mathrm{b}, \mathrm{t}, \mathrm{w})$

where

$$
\mathrm{g}(\mathrm{b}, \mathrm{t}, \mathrm{w})=\frac{1}{\mathrm{Z}_{0}}\left[\frac{\delta \mathrm{Z}_{0}}{\delta \mathrm{b}}-\frac{\delta \mathrm{Z}_{0}}{\delta \mathrm{w}}-\frac{\delta \mathrm{Z}_{0}}{\delta \mathrm{t}}\right]
$$

where

$Z 0 \ldots$ is the characterestic impedance.

$f$.... is the frequency in $\mathrm{Ghz}$

$\mu_{0} \ldots$ is the permeability

$\sigma \quad \ldots$ is the conductivity of the conductor

$b, t, w \ldots$ are the strip line dimensions in $\mathrm{cm}$

By substituting equation (8) and (9) into equation (5) it is possible to write $\frac{1}{\mathrm{Q}_{0}}=\frac{1.5911}{\sqrt{\sigma \mathrm{f}}} \mathrm{g}(\mathrm{b}, \mathrm{t}, \mathrm{w})+\tan \delta$

Plotting $\frac{1}{Q_{0}}$ versus $g(b, t, w)$ results in a ștraight tine with a slope $m=\frac{1.5911}{\sqrt{\sigma \mathrm{f}}}$, and a $y$ intercept $\mathrm{b}=\tan \delta$. This gives a method for separating the loss terms by producing multiple stripline resonators and plotting their unloaded quality factors versus $g(b, t, w)$ We can get a complete characterization for the used material in our stripline resonator by calculating the relative permitivity. This can be done by measuring $\$ 21$ using the frequency domain measurements from which we can get the resonance frequencies of the stripline resonator and using equation (4) we can calculate the relative permitivity of the dielectric material at these frequencies.

\section{PROPER DESIGN OF RESONATOR GAP}

For the coplaner coupled stripline configuration under consideration in this work, the gap is defined as the transition / isolation region between the coplaner line and the stripline. The gap dimensions are expected to affect both the shape of the stripline resonances (and hence the quality factor $\mathrm{Q}$ ) as well as the frequencies ( $\mathrm{fO}$ ) at which they occur. In other words, the gap dimensions affect both $\mathrm{f} O$ and $\mathrm{Q}$, which are the two primary values in determining the complex permitivity of the dielectric material. This makes it critical to properly design the gap transition region and choose its optimum dimensions. 
In order to have a complete appreciation of the effect of the gap dimensions on the response of the stripline resonator, we need to simulate a set of stripline resonators with different gap dimensions. From the results of these simulations, we will be able to put the suitable modifications to the design of the stripline resonator by controlling the gap dimensions.

The S21 vs. frequency results of these simulations shows that:

A transition region with a large gap would cause the coupling to be very week to observe resonance.

-A transition region with a narrow gap would increase external loading effects that degrade the quality factor of resonance, also decrease the frequency range of operation of the resonator

- The capacitive behavior of the gap turns into more complex one above some certain frequency depending on the dimensions of the gap, as we will see in the next section from the Pspice model of the gap.

-A relatively small overlap would help improve the behavior of the gap at higher frequencies.

\section{PSPICE MODELING OF THE RESONATOR GAP}

As we had mentioned before, the capacitive behavior of the gap turns into more complex one above some certain frequency depending on the dimensions of the gap. In this section we will try to verify that observation, using the Pspice software. Using the S21 values from IE3D simulation of transition region, we can drive a lumped element model of the gap using Pspice software.

We will model the transition region using the Pspice software including a part from the coplaner line, the overlap (coupling region), and a part from the center stripline. The result of the Pspice modeling is given in Figure (3). This model is derived by trial and error while comparing S21 of the Pspice model to that of the IE3D simulation. The S21 comparison corresponding to the model of Figure (3) is given in Figure (4). It is seen from the figure that a good agreement in S21 is achieved indicating good modeling accuracy.

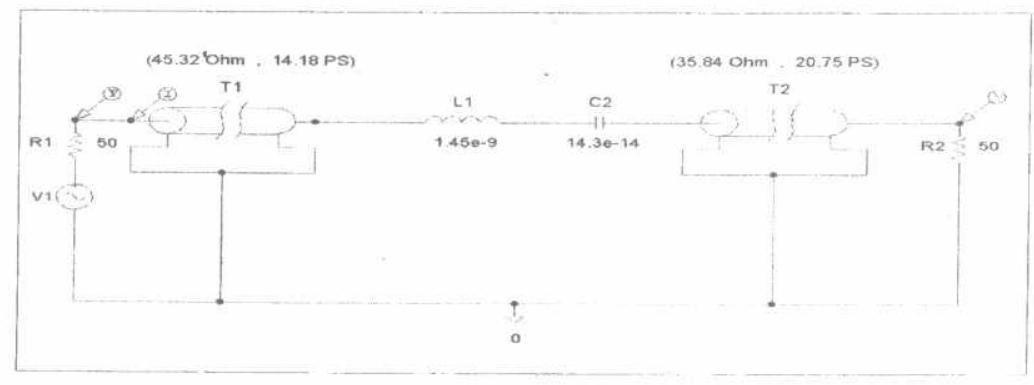

Fig (3) Pspice model for a transition of 0 mils overlap 


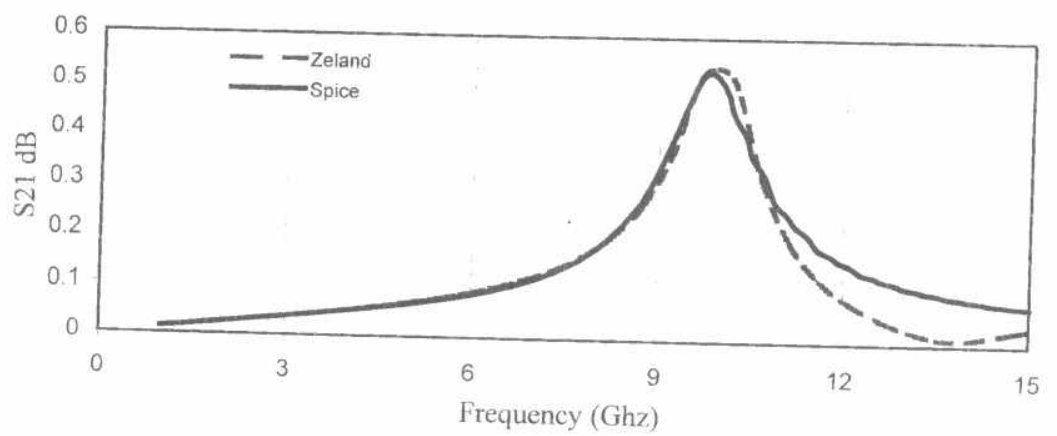

Fig (4) Frequency response of Pspice modeling for a transition of 0 mils overlap As we can see from Figure (3), the transition region consists of three parts. The 1st part is a transmission line representing the coplaner line part, followed by the lumped representing the of the coupling region, followed by the other transmission line coupling region takes the lime part. It is worth noting that the lumped model of the frequencies the $C$ part of this moximate form of a series $L-C$ combination. At the lower gap. However, at the upper frequencies, thes and hence the capacitive behavior of the inductive coupling. The impedance of the the $L$ dominates over the $C$ resulting in an frequency range resulting in minimize the loading effect of the source and ling to and from the stripline resonator to The L- C combination, and hence the

c 1 comibit a low impedance around a certain frequency at which $\omega \mathrm{L}=\frac{1}{\omega \mathrm{C}}$ reaches its peak value for a given strit this frequency and in its vicinity, the coupling resonator and thus a lowered dielectric properties looses its accuracy In this frequency range, measurement of the inaccuracies in determining the resonant frause the lowered $Q$ values, which means determining the $\mathrm{Qc}$ and $\mathrm{Qd}$ terms in ans in equation (5)

sought to be added to the transition coupling behavior, reactive tuning elements are the low impedance behavior and weaken the goal is to add reactance(s) to tune out resonator section.

We found that we can improve the behavior of the gap and the overall resonator response at higher frequencies by adding a overall resonator shown in Figures (5) and (6). 


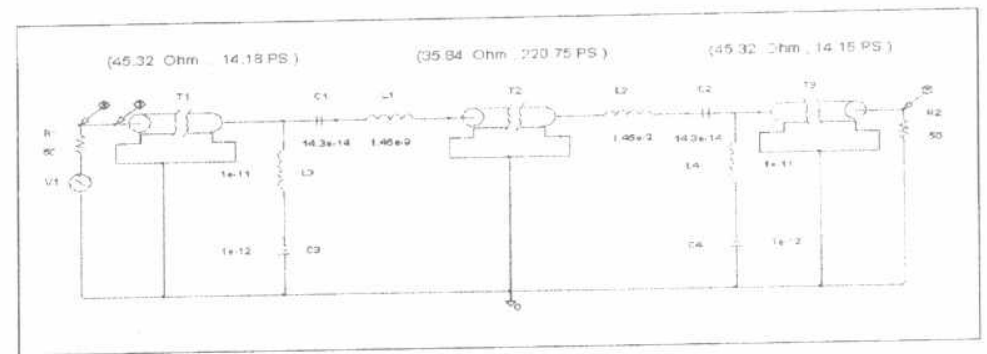

Fig (5) Overall Pspice model with compensating shunt branch

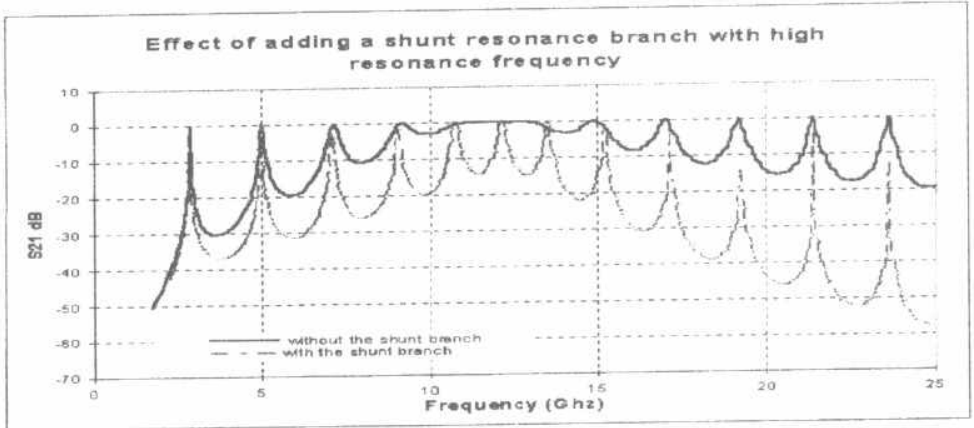

Fig (6) Overall response with shunt resonance branch: overlap -40 mils

\section{CONCLUTION}

In conclusion to this paper, we can say that the coplaner coupled stripline resonator is an accurate technique in measuring the electric characteristics of the dielectric material at the microwave frequency range except at some frequencies (depending on the dielectric material) at which the L- C combination, and hence the coupling region exhibit a low impedance around a certain frequency at which $\omega \mathrm{L}=\frac{1}{\omega \mathrm{C}}$

At this frequency and in its vicinity, the coupling reaches its peak value for a given stripline resulting in excessive loading to the resonator and thus a lowered $Q$ value. In this frequency range, measurement of the dielectric properties looses its accuracy because the lowered $Q$ values, which means inaccuracies in determining the resonant frequencies as well as great error in determining the Qc and Qd terms in equation (5). 
Proceedings of the $9^{\text {th }}$ ASAT Conference, 8-10 May 2001 Paper BT-02 846

Also, we can say from the lumped element model of the gap that, the gap behavior is not purely capacitive in the high frequency range, but it is more complex one. Also we can improve the transition and the overall stripline resonator frequency response in the high frequency range by adding a suitable compensating resonant shunt branch to our model of the gap.

\section{REFERENCES}

[1] K.C.Gupta, (1979) "Microwave" Wiley Eastern Limited New Delhi.

[2] David M.Pozar (1990) "Microwave Engineering" Addison-Wesley Publishing Company, Inc.

[3] S.B. Cohn, "Characteristic Impedance of the Shielded-Strip Transmission Line," Trans. IRE, vol. MTT-2, no. 2, pp. 52-57, July 1954.

[4] Mohammed A. Saed, "Measurements of the Complex Permittivity of Low-Loss Planar Microwave Substrates Using Aperture-Coupled Microstrip Resonators," IEEE

Transaction on Microwave Theory and Techniques, vol. 41, no. 8. August 1993.

[5] Wansheng Su, Sedki M. Riad, and Aicha Elshabini-Riad, "Microwave Material Characterization Using Stripline Resonators," The International Journal for Hybrid Microelectronics, vol. 14, no. 2, June 1991.

[6] Seymour B. Cohn, (1955) "Problems in Strip Transmission Lines", IRE Transactions on Microwave Theory and Techniques, Vol. MTT-3, March.

[7] Matthaei, George, L.; Young, Leo; and Jones, E.M.T. (1964) "Microwave Filters Impedance Matching Networks, and Coupling Structures" McGraw-Hill, New York. 